\title{
Notas sobre Hildete Pereira de Melo y Débora Thomé (2018). Mulheres e Poder. Historias, ideias e indicadores. Rio de Janeiro: FGV Editora. 190 páginas
}

\author{
Julieta Mulet \\ mulet.julieta@gmail.com \\ Universidad Católica de La Plata, Argentina
}

Recepción: 16 Octubre 2019

Aprobación: 01 Septiembre 2020

Publicación: 01 Marzo 2021

Cita sugerida: Mulet, J. (2021). [Revisión del libro Mulheres e Poder.

Histórias, ideias e indicadores por H. Pereira de Melo y D. Thomé].

Descentrada, 5(1), e143. https://doi.org/10.24215/25457284e143

La obra Mulheres e Poder. Histórias, ideias e indicadores es producto de la percepción de una necesidad académica y a la vez práctica: el poder atraviesa transversalmente la existencia humana, e indagar dónde se encuentran las brechas que históricamente y hasta nuestros días mantienen alejadas a las mujeres del mismo, constituye un paso fundamental para pensar y transformar nuestras realidades.

La escasa sistematización de los estudios realizados en torno a las mujeres y su relación con el poder a lo largo del tiempo contrasta con los abundantes esfuerzos de producción de conocimiento en la materia, y es precisamente la conciencia de este contraste el motor que impulsa el trabajo de las autoras, quienes se proponen condensar múltiples debates y ofrecer información variada en un volumen de amena lectura para aquellas personas que se inician en el abordaje de estas temáticas y para quienes deseen profundizar en ellas.

Que la producción académica existente sobre el binomio mujeres-poder se encuentre dispersa, da cuenta de una realidad que nos interpela: la ciencia es uno de los tantos espacios de los cuales las mujeres han sido excluidas y, junto a ellas, han sido dejados de lado en las agendas de investigación los enfoques que ponen en relieve sus problemáticas específicas. ¿Significa esto que las mujeres no participan en espacios de construcción del conocimiento? De ningún modo. Sin embargo, reconocer y problematizar el hecho de que el campo científico legitimado ha sido históricamente blanco y masculino, producto de las relaciones de poder 
dominantes, constituye un primer paso para avanzar en la conquista de espacios en este terreno, en el que se disputa la construcción de sentidos y saberes.

El potencial del escrito como llamado a la reflexión, pero también a la acción, está íntimamente vinculado a las experiencias de vida de las autoras como académicas y activistas feministas. Hildete Pereira de Melo es economista, ejerció funciones de coordinación y asesoramiento en materia de políticas públicas con perspectiva de género en el área de Educaçao y Cultura y en la Secretaria de Politicas para as Mulheres, y desde los años '70 participó de varias de las luchas relatadas en el libro. Por su parte, Débora Thomé es politóloga, periodista y militante feminista y ha participado en la formación de candidatas en un curso financiado por la Secretaria de Mulheres.

En un contexto de creciente importancia ganada por los movimientos feministas en la región, el libro de Hildete Pereira de Melo y Débora Thomé tiene una finalidad teórico-práctica y viene a renovar el interés sobre el vínculo de las mujeres y el poder, a lo largo de ocho capítulos en los que recorren las trayectorias teóricas de los feminismos, el lugar que han ocupado las mujeres en la historia del mundo en general, y de Brasil en particular, y su relación con la distribución social del poder en el ámbito demográfico, educativo, laboral, político, legislativo y de las políticas públicas en este país del cono sur.

Así, en el primer capítulo, Pereira de Melo y Thomé narran la evolución de las principales formulaciones teóricas del campo disciplinar de los estudios de género al calor de las diversas formas adoptadas por las luchas feministas, cuyo recorrido es identificado por las autoras como una historia de victorias y derrotas (Pereira de Melo y Thomé, 2018).

La emergencia de las mujeres en la arena política fue la antesala de las discusiones académicas, y qué es el feminismo fue la pregunta que articuló los debates. Retomando esa cuestión, las autoras plantean al feminismo en tanto conjunto de movimientos que abogan por la igualdad de derechos a través de la liberación de la opresión de las mujeres por los hombres (Pereira de Melo y Thomé, 2018) y, partiendo del que consideran el texto fundador del feminismo del siglo XX, El segundo sexo de Simone de Beauvoir (1949), desarrollan el discurrir de las diversas corrientes teóricas, sus antecedentes y principales exponentes, en un recorrido que va desde el feminismo liberal y el radical, al feminismo marxista, cultural y negro, llegando a abarcar visiones más actuales como el feminismo interseccional. El final del capítulo recupera el concepto de género en tanto herramienta de análisis y pone de manifiesto las principales líneas de debate (Scott, 2008; Haraway, 1988; Butler, 2007).

El segundo capítulo coloca en perspectiva el papel de las mujeres en la historia mundial y, sobre todo, la importancia de sus luchas colectivas como manifestación de resistencia y disputa a los poderes dominantes esencialmente patriarcales. De este modo, pasando desde las acciones de las primeras feministas y su participación en las revoluciones del siglo XVIII, cuyo objetivo de subvertir el orden patriarcal establecido quedó trunco, hasta los reclamos por el reconocimiento de los derechos políticos, las autoras se proponen hacer oír las voces silenciadas por la pluma de quienes escribieron la historia, en un intento por reintegrar a las mujeres a la misma (esto es, su rescate en tanto sujetas históricas) y por restituirles su propia especificidad dentro de la disciplina (Ramos Escandón, 1997).

Como en el resto del mundo, la historia de Brasil también confinó a sus mujeres al olvido, siendo ese el eje temático del tercer capítulo. Las dificultades provistas por la falta de fuentes motivan el recorte temporal de las autoras al período postcolonial, dejando así fuera del análisis una parte central de la historia de la opresión de las mujeres en un país que tuvo la excepcionalidad de ver la convivencia territorial de la metrópoli y la colonia y que, una vez alcanzada su independencia, adoptó forma de imperio para décadas más tarde ser el último de la región en abolir la esclavitud. Así, las autoras retoman las embrionarias luchas feministas de la segunda mitad del siglo XIX, en su mayor parte encabezadas por una elite femenina blanca e intelectual, y los movimientos, mucho más diversos, iniciados a partir de la década del “ 40 del siglo pasado. Recuperar a las olvidadas de la amnesia sexista de la academia no es una labor sencilla. A riesgo de personalizar los procesos y diluir lo colectivo, las autoras deciden atravesar las vivencias, siempre subjetivas, de las mujeres que fueron 
clave en las luchas -no desprovistas de dificultades, pero ganadas- por la abolición de la esclavitud, los derechos políticos y el acceso a la educación, como así también aquellas que fueron pilares de la organización de la resistencia al autoritarismo y la vuelta a la democracia durante el siglo XX.

En el cuarto capítulo, Pereira de Melo y Thomé se proponen desentrañar cuestiones demográficas que les permiten afirmar que el colectivo de mujeres al que con frecuencia se da un tratamiento de "minoría" no constituye una minoría numérica, sino que tal categoría le es adjudicada como resultado de la distribución desigual del poder en la sociedad. De este modo, las autoras proveen datos estadísticos y análisis resultantes del cruce de la información recabada sobre la población de Brasil en materia de fecundidad, natalidad y mortalidad materna, escolarización, urbanización y migraciones. Ello abre un sinfín de interrogantes en torno a la reconfiguración de los roles en el marco de la familia, el cuestionamiento de la maternidad obligatoria y el ejercicio de la sexualidad, el papel que ha de asumir el Estado frente a los cambios en la composición poblacional y la necesidad de adecuación de las políticas públicas a las nuevas realidades, incluyendo debates tales como la educación sexual integral, el embarazo adolescente y la legalización del aborto.

La educación, herramienta fundamental para identificar las desigualdades sociales y bastión de la emancipación de las mujeres en Brasil según las autoras, constituye el eje temático del quinto capítulo, que logra combinar información cuantitativa junto al desarrollo histórico de la lucha de las mujeres por el acceso a este espacio. Así, Pereira de Melo y Thomé, evalúan el proceso educacional de las mujeres en su país, sobre todo en el acceso a la educación superior y los campos del saber en los que se desarrollan las mujeres, muchas veces vinculados a disciplinas socialmente asociadas a la construcción de lo femenino como prolongación de la maternidad, considerando, además de la cuestión de género, otras categorías analíticas como la clase y la etnia.

La lucha de las mujeres por la educación en Brasil es otra batalla ganada por los feminismos y, actualmente, la población femenina escolarizada supera numéricamente a la masculina. No obstante, la lucha tiene continuidad frente al sexismo que atraviesa al mercado de trabajo. El capítulo seis pretende dar cuenta de ello, dejando en evidencia las dificultades que enfrentan las mujeres en el acceso a puestos jerárquicos en su vida laboral pero, por sobre todo, acentuando el déficit de la mayoría de los estudios económicos que ponen la vista en los ámbitos de producción asalariada, dejando a un lado trabajos reproductivos y de cuidado ejercidos mayoritariamente por las mujeres. Así, ese trabajo doméstico no remunerado, realizado por las mujeres y oculto tras la fachada de amor familiar, refuerza la noción binaria y sexista de la división sexual del trabajo (hombre-proveedor/mujer-reproductora) que excluye a las mujeres del circuito productivo valorado en el marco del sistema capitalista dominante y que no es más que otra dimensión de la relación de dominación que los hombres ejercen sobre las mujeres.

La reclusión de las mujeres en el hogar las alejó por mucho tiempo de la esfera pública y, con ello, de los ámbitos de participación y representación política, tema al que se refieren las autoras en el capítulo siete. Aún tras la conquista del derecho a votar y ser votadas en 1932, las mujeres brasileñas continuaron enfrentando enormes dificultades en el acceso al poder político, que siguen existiendo en nuestros días dado que disputar estos espacios implica desafiar la estructura de poder masculina vigente. Ni la sanción de la ley de cuotas de género para los cargos legislativos ni la experiencia de ejercicio del más alto cargo ejecutivo de la Nación por una mujer, logró accionar como motor para comenzar a revertir esta situación: las mujeres siguen siendo una escasa minoría dentro del Parlamento ${ }^{1}$ y la presidencia de Dilma Rousseff fue interrumpida tras su destitución del cargo de presidenta en 2016 luego de un cuestionable proceso de impeachment que constituyó un duro golpe a la estabilidad de la democracia brasileña y puso de manifiesto la misoginia del poder político y judicial.

Las autoras esbozan una correlación lineal entre la participación y la representación que puede ser puesta en cuestión, por cuanto que la presencia de mujeres en cargos públicos no implica per se la representación de los intereses del colectivo de género. No obstante, es importante reconocer que la posibilidad de participar del juego político abre espacio a que mujeres feministas puedan acceder a los poderes institucionalizados 
(Altamirano y Mulet, 2019). En esos términos, y desde una perspectiva propia de la Ciencia Política, Pereira de Melo y Thomé retoman las nociones de representación descriptiva, sustantiva y simbólica en que puede dividirse el concepto de representación (Pitkin, 1985; Franceschet y Piscopo, 2008; Caminotti, 2013). Resulta interesante la inclusión de los efectos simbólicos derivados de la ocupación de espacios de poder por las mujeres, siendo este un aspecto poco explorado y pasible de ser pensado como manifestación de la representación descriptiva y sustantiva, más que como una dimensión en sí misma del concepto de representación política.

Que la representación se haga lugar en la realidad material traduciéndose en legislación y políticas públicas con perspectiva feminista depende del reconocimiento de las desigualdades existentes para trabajar sobre ellas (Pereira de Melo y Thomé, 2018). Que el feminismo pueda ocupar lugares de toma de decisión y a la vez que actúe como grupo de presión, es fundamental para alcanzar ese horizonte de posibilidades de transformación. En el capítulo ocho, las autoras recalcan que ningún derecho fue ganado sin una fuerte actuación de las mujeres y repasan los principales hitos que, a lo largo de la historia de Brasil, fueron permitiendo construir la ciudadanía de las mujeres, entre ellos: el Estatuto da Mulher Casada, la Lei do Divórcio, el Programa de Assistência Integral à Saúde da Mulher, el Conselho Nacional dos Direitos da Mulher, el reconocimiento de la igualdad de derechos y obligaciones de hombres y mujeres en la Constitución de 1988. Entre 2003 y 2010 , período que reconocen como el de mayor producción de políticas de género, cabe señalar sucesos como la Lei Maria da Penha para la prevención y combate de la violencia contra la mujer, la creación de la Secretaria Especial de Politicas para as Mulheres, el Plano Nacional de Políticas para as Mulheres y la Lei do Feminicídio, entre otros.

Ante la incertidumbre que supone el futuro en lo que a las políticas de género se refiere, la defensa de los derechos conquistados se convierte en un deber. Arrojar luz sobre aquello que permaneció tanto tiempo en penumbras y dar voz a las silenciadas como insumo para la acción, es una declaración de intenciones de las autoras. A su vez, la decisión metodológica de sacrificar profundidad en favor del abordaje de una multiplicidad de temas desde una perspectiva de género, lo cual supone al mismo tiempo una toma de posición teórica y epistemológica, permite a las autoras cumplir con su objetivo de brindar una herramienta para analizar desde Latinoamérica el reparto del poder en las sociedades y el lugar que ocupan las mujeres en esa ecuación: cómo sus vidas son atravesadas por el poder, cómo lo disputan, cómo lo ejercen y, también, cómo le oponen resistencia.

Esta obra constituye, así, un valioso aporte en tanto estado del arte de la cuestión para (re)pensar las construcciones académicas sobre las mujeres y el poder, elevando el feminismo a la categoría de teoría política en tanto se refiere a la dinámica del poder y su distribución desigual en las sociedades. Cabe mencionar que la visión compartida por las autoras se inscribe en una perspectiva binaria que, como tal, deja fuera del análisis otras identidades sexogenéricas a las que, sin embargo, reconocen como parte activa y sustantiva de las luchas feministas, lo que nos coloca en posición de preguntarnos sobre la configuración diversa y plural del sujeto político de los feminismos actuales y el desafío de escribir sobre ello.

En ese sentido, Hildete Pereira de Melo y Débora Thomé invitan a cuestionarnos el mundo en el que vivimos, para transformar el conocimiento en lucha colectiva para el ejercicio pleno de los derechos que las mujeres supieron conseguir y el logro de los que aún les son vedados, de cara a hacer de nuestras democracias sistemas más justos, participativos, representativos e inclusivos.

\section{REFERENCIAS}

Altamirano, J. y Mulet, J. (2019). Cuota de género y sistemas electorales: Argentina y Brasil en perspectiva comparada. Descentrada, 3(2).

Butler, J. (2007). El género en disputa. El feminismo y la subversión de la identidad. Barcelona: Paidós. 
Caminotti, M. (2013). La representación política de las mujeres en el período democrático. Revista SAAP. Publicación de Ciencia Politica de la Sociedad Argentina de Análisis Politico 7(2), 329-337.

Franceschet, S. y Piscopo, J. (2008). Gender Quotas and Women's Substantive Representation: Lessons from Argentina. Politics \& Gender (4)3, 393-425.

Haraway, D. (1988). Situated Knowledges: The Science Question in Feminism and the Privilege of Partial Perspective. Feminist Studies, 14(3), 575-599.

Pereira de Melo, H. y Thomé, D. (2018). Mulheres epoder. Histórias, ideias e indicadores. Río de Janeiro: FGV Editora. Pitkin, H. F. (1985). El concepto de representación. Madrid: Centro de Estudios Constitucionales.

Ramos Escandón, C. (1997). La nueva historia, el feminismo y la mujer. En Género e Historia: La historiografía sobre la mujer. México: Instituto Mora.

Scott, J. (2008). El género: una categoría útil para el análisis histórico. En Género e historia. México: Fondo de Cultura Económica.

\section{Notas}

1 Para el periodo 2015-2019, la Cámara de Diputados contó con 62 diputadas de un total de 513 miembros y el Senado con 13 senadoras de 81 miembros total (Altamirano y Mulet, 2019). Cabe aclarar que las cuotas por sí solas no garantizan el acceso al poder puesto que en ello influyen el sistema electoral, el sistema de partidos, la propia estructura de las cuotas y, por supuesto, el voto popular. 